\title{
Asma bronquial infantil: aspectos clínicos y de laboratorio
}

\author{
DRS.: GONZALO GOMEZ *, SERGIO IBANEZ *, VICTOR LAMA *
}

INTRODUCCION :

El asma bronquial se puede definir como un estado caracterizado por una obstrucción parcial de la vía aérea, reversible con el tiempo, ya sea espontáneamente o como resultado de tratamiento (1).

Es indudable que se asiste a un aumento en la prevalencia de esta afección (1). Frandsen encuentra 1,6\% en Dinamarca (2). Rappaport encuentra 10\% en Estados Unidos. Es causa de un alto ausentismo escolar (3) y es motivo de múltiples consultas en las policlínicas de pediatría.

Hay que señalar como hecho etiopatogénico fundamental a un estado de hiperreactividad bronquial, que en la gran mayoría de los niños asmáticos se asocia a un terreno atópico. Sobre este sustrato actúan múltiples factores como desencadenantes de las molestias asmáticas. Cuando hablamos de terreno atópico, nos estamos refiriendo a un defecto inmunológico fundamentado en un estado alterado del terreno que reconoce un rasgo genético. Las características clínicas de la diátesis atópica han sido largamente descritas $(4,5,6,7)$; los aspectos inmunológicos del atópico incluyen tanto la tendencia a desarrollar hipersensibilidad de tipo I mediado por IgE (Reaginas), a través de una vía natural, a sustancias del medio ambiente usualmente inocuas para el común de los seres humanos; como también deficiencias de IgA en el período de lactantes $(1,8$, $9,11,12,13,14,15)$.

Como ya se mencionó, el hecho clave etiopatogénico sería el de un estado de hiperreactividad bronquial propia del terreno asmático, probablemente por una alteración

* Servicio de Enfermedades Broncopulmonares, Hospital Luis Calvo Mackenna. Sede Oriente, Univ. de Chile. de la función beta adrenérgica. (1, 4, 10, 11, $14,16,17,19,20)$. Como factores que gatillan la hiperreactividad bronquial han sido involucrados varios: alérgenos, mediadores químicos, factores físicos, psíquicos, ejercicios, infección respiratoria $(1,4,11,12,13$, $16,21,25$ ).

En la presente publicación nos referimos al análisis clínico y de laboratorio de $108 \mathrm{ni}-$ ños asmáticos. Una comunicación relativa a resultados terapéuticos se publicará próximamente.

Material y MEtodo. Se estudian 108 casos con Asma perenne, ingresados a la policlínica de broncopulmonares desde el 15 de junio de 1969 al 15 de junio de 1973, con un tope de edad de 12 años, con radiología de tórax normal, y sin patología cardiovascular asociada.

A todos los pacientes se les practicó anamnesis dirigida, radiografía de tórax y hemograma. Examen parasitológico de deposiciones en todos aquellos casos con eosinofilia mayor de $4 \%$.

A todos los casos se les practicó test intradérmico con polvo de habitación, mezcla de hongos, y mezcla de pólenes a concentración de 1.000 P.N.U. por mililitro, del Laboratorio Center, de acuerdo a las especificaciones de dicho laboratorio en cuanto a medición, y usando como etalon un control intradérmico de solución salina fisiológica fenolada al $0,5 \%$.

RESUlTADOS. 1) Distribución por sexo: la tabla № 1 nos señala un predominio del sexo masculino con un $59,2 \%$.

2) Edad al momento del Ingreso: la tabla No 2 nos muestra que estos pacientes con- 
T A B L A N $\mathrm{N}: 1$

DISTRIBUCION POR SEXO

\begin{tabular}{lcc}
\hline & No de casos & $\%$ \\
\hline Masculino & 64 & 59,2 \\
Femenino & $\frac{44}{40,7}$ \\
& $\frac{100}{108}$ & 100 \\
\hline
\end{tabular}

T A B L A N $: 2$

EDAD AL MOMENTO DEL INGRESO

\begin{tabular}{lr}
\hline-2 años & 1 \\
2 a 5 años & 53 \\
6 o más & 54 \\
& 108 \\
\hline
\end{tabular}

curren a un centro especializado después de los 2 años de edad, y llama la atención que la mitad de los casos lo hace después de los 6 años.

3) Edad de comienzo de las molestias asmáticas: Tabla No 3 . Vemos que un $40 \%$ de los casos inician su asma antes de los 2 años y que sólo un $22 \%$ lo hace después de $\operatorname{los} 6$ años.

$$
\text { T A B L A } \mathrm{N}: 3
$$

EDAD DE APARICION DE LAS MOLESTIAS ASMATICAS

\begin{tabular}{lll}
\hline & No & $\%$ \\
\hline-2 años & 44 & 40,7 \\
2 a 5 años & 40 & 37 \\
6 o más & 24 & 22,2 \\
& 108 & 100
\end{tabular}

4) Tiempo de evolución de la enfermedad al momento del ingreso: La tabla № 4 nos muestra, que existe un $38,8 \%$ de pacientes que concurren a un centro especializado después de 3 años de haber iniciado sus molestias asmáticas.
$\begin{array}{llllllll}\mathrm{T} & \mathrm{A} & \mathrm{B} & \mathrm{L} & \mathrm{A} & \mathrm{N} & ?\end{array}$

TIEMPO DE ENFERMEDAD AL INGRESO

\begin{tabular}{llc}
\hline & $\mathrm{N}^{\circ}$ & $\%$ \\
\hline-1 año & 25 & 23,1 \\
1 a 2 años & 41 & 37,9 \\
3 o más & 42 & 38,8 \\
\cline { 2 - 3 } & 108 & 100 \\
\hline
\end{tabular}

5) Antecedentes Personales y Familiares: Tabla № 5 . Se observa que un $72,2 \%$ de nuestro material tenía antecedentes familiares marcados de patología alérgica, y un $62 \%$ relató antecedentes familiares marcados de Asma bronquial. Hay que recalcar que un $45,3 \%$ de los pacientes sufrió durante el primer año de vida de cuadros bronquiales obstructivos (dos o más), diagnosticados en su oportunidad como bronquiolitis, bronquitis espástica, bronquitis obstructiva, etc.

T A B L A $\mathrm{N}: 5$

ANTECEDENTES FAMILIARES $Y$ PERSONALES: 108 CASOS

\begin{tabular}{lll}
\hline & $\mathrm{N}^{\mathrm{a}}$ & $\%$ \\
\hline FAM. Alergia & 78 & 72,2 \\
FAM. Asma & 67 & 62 \\
Personales Alergia Piel & 41 & 37,9 \\
Pers. Bronq. obst. repetidas & 49 & 45,3 \\
lact. & & \\
\hline
\end{tabular}

T A B L A $N \div 6$

FACTORES DESENCADENANTES CRISIS ASMATICA: 108 CASOS

\begin{tabular}{lrr}
\hline & Na & $\%$ \\
\hline Infección respiratoria & 104 & 96,2 \\
Ejercicio & 48 & 44,4 \\
Desconocido & 41 & 37,9 \\
Físicos & 33 & 30,5 \\
Emociones & 4 & 3,7 \\
\hline
\end{tabular}


TEST ALERGIA POSITIVO

\begin{tabular}{lcccccc}
\hline Intensidad Test. & $\mathrm{N}$ ( & $\%$ & $\begin{array}{c}\text { menos de } 6 \text { años de edad } \\
\mathrm{N}^{\circ}\end{array}$ & $\begin{array}{c}6 \text { o más años } \\
\mathrm{N}^{\circ}\end{array}$ & $\begin{array}{c}\text { \% } \\
\%\end{array}$ \\
\hline$+/++$ & 58 & 57,4 & 37 & 78,7 & 21 & 38,8 \\
$+++/++++$ & 43 & 42,5 & 10 & 21,2 & 33 & 61,1 \\
\hline TOTAL & 101 & 100 & 47 & 100 & 54 & 100 \\
\hline
\end{tabular}

6) Factores desencadenantes de las crisis asmáticas: Tabla $\mathrm{N}^{\circ}$ 6. Se aprecia que el factor más invocado sería la infección respiratoria. En un 37,9\% no se logró precisar factor alguno. Factores emocionales fueron detectados en 4 casos, (todos los cuales fueron mayores de 6 años de edad).

7) Test de alergia positivo: en 101 del total, lo cual representa un $93,5 \%$ de positividad. La Tabla No 7 nos muestra la distribución según la intensidad de la reacción intradérmica y por grupo de edad. Cada cruz equivale a $5 \mathrm{~mm}$.

8) Test de Alergia positivo: distribución porcentual por alergenos. Tabla No 8 . Notamos que en general la inmensa mayoría de los casos manifiesta positividad a polvo de habitación, tanto en menores como en mayores de seis años de edad. El \% de positividad a hongos y muy especialmente a pólenes se hace mayor a medida que aumenta la edad en que se efectuó el test. Hay que destacar, que en este trabajo no se consideraron los casos de Asma polínico estacional. Los porcentajes según se aprecia en la presente tabla, se ven más abultados en el grupo de niños de seis o más años, esto

$$
\begin{array}{lllllll}
\text { T A B L A } & \mathrm{N}: 8
\end{array}
$$

TEST DE ALERGIA POSITIVO 101 CASOS: DISTRIBUCION POR CIENTO ALERGENOS Y POR EDAD

En el total Por edad

\begin{tabular}{lccc}
\hline & $\%$ & -6 años $\%$ & $60+$, años \% \\
\hline Polvo Habit. & 91,08 & 85,10 & 96,20 \\
Hongos & 64,35 & 57,44 & 70,37 \\
Pólenes & 48,51 & 36,17 & 59,25 \\
\hline
\end{tabular}

tanto por un aumento de la positividad a cada alergeno en particular, como a un aumento de la incidencia de positividad a dos o más alergenos a la vez en un mismo paciente.

9) Eosinofilia: se descartaron aquellos casos portadores de enteroparasitosis $u$ otras causas, quedando 71 pacientes. Hay que hacer presente que en 200 hemogramas tomados al azar en el departamento de hematología de nuestro hospital, un $70 \%$ presentaba un número de eosinófilos normales, un $22 \%$ de 5 a $10 \%$ y un $8 \%$ tenía eosinofilia de $11 \%$ o más.

La tabla No 9 nos muestra los porcentajes de eosinófilos encontrados en nuestro material, y la relación de éstos con la intensidad del test cutáneo de alergia. Apreciamos que una cantidad de eosinófilos dentro de límites normales sólo se encontró en un $26,7 \%$ de los casos y que 11 o más eosinófilos fueron detectados en 35,21\% de los casos. Por otra parte relacionando los resultados con el test de alergia, vemos que el grupo de pacientes que manifestó reacciones intradérmicas intensas el test cutáneo de alergia sólo en un $10,6 \%$ de los casos el $\mathrm{N}$ \% de eosinófilos estuvo en los límites normales, y $50 \%$ de estos casos presentó eosinofilia de 11 o más. Esta tendencia fue menos acentuada en el grupo de pacientes con reacciones intradérmicas débiles.

\section{COMENTARIO Y CONCLUSIONES}

Del estudio de nuestro material se pudo constatar un predominio del sexo masculino, hecho muy documentado en la literatura $(1,21,22)$.

Hay que destacar el hecho de que en nuestra casuística un número importante inicia sus molestias asmáticas antes de los 2 años de edad, y que la mayoría lo hace antes de los seis años; esto está en concordancia con 
T A B L A N : 9

EOSINOFILIA EN 71 HEMOGRAMAS RELACION CON INTENSIDAD DEL TEST

\begin{tabular}{|c|c|c|c|c|c|c|}
\hline \multirow{2}{*}{$\begin{array}{l}\text { ó de } \\
\text { eosinófilos }\end{array}$} & \multirow{2}{*}{ No } & \multirow[b]{2}{*}{$\%$} & \multicolumn{4}{|c|}{$\begin{array}{c}\text { Test de alergia } \\
+1++1+++1++++\end{array}$} \\
\hline & & & $\mathrm{N}^{\circ}$ & $\%$ & & $\%$ \\
\hline-5 & 19 & 26,76 & 13 & 35,13 & 6 & 10,64 \\
\hline $5-10$ & 27 & 38,02 & 16 & 43,24 & 11 & 32,35 \\
\hline 11 o más & 25 & 35,21 & 8 & 21,62 & 17 & 50,00 \\
\hline TOTAL & 71 & 100 & 37 & 100 & 34 & 100 \\
\hline
\end{tabular}

lo descrito por diversos autores $(22,23)$. Llama la atención la tardanza que existe en que estos niños concurran a un centro especializado.

Hemos encontrado en un alto porcentaje de los pacientes, antecedentes familiares de alergia y/o asma, así como de antecedentes personales de afecciones alérgicas de la piel $(1,5,6,7,24)$.

El hecho de que en nuestra casuística $45 \%$ de los casos acusaron el antecedente de cuadros bronquiales obstructivos en el primer año de vida, nos hace pensar por una parte que podría tratarse en muchos de estos casos de inicio de su asma, o que estos niños siendo portadores de un terreno atópico tendrían en ese período de su vida una deficiencia inmunológica ( $\operatorname{IgA})$, que favorecería a determinadas infecciones del aparato respiratorio $(15,25,26)$.

Hay que destacar la dificultad de determinar los factores desencadenantes de las crisis en la anamnesis, sobre todo en niños menores de seis años; pero es evidente el importante papel que juega la infección respiratoria $(1,3,5,21,22,24)$.

En cuanto al test de alergia, llama la atención al alto porcentaje de positividad, 101 de 108 casos, en contraposición al asma de los adultos. Hay que destacar que en los 7 casos que resultaron con test negativos, retesteados después de un año, en 4 el resultado fue positivo $(1,22,24,27)$. Es evidente que la capacidad de manifestar reacciones intensas intradérmicas frente a los alergenos está en relación directa con la edad del paciente. Se podría concluir que el asma "intrínseco" sería de escasa frecuencia en el niño.

En cuanto a la distribución de los alergenos en los test positivos, llama la atención el alto porcentaje de positividad a hongos en nuestro material, en segundo lugar des- pués de polvo de habitación. En lo que se refiere a positividad a polénes, vemos que los porcentajes se incrementan con la edad; estos porcentajes podrían ser algo mayores ya que en nuestra casuística no se incluyeron los asmáticos polínicos estacionales (1, $7,22,23,27)$.

Por último se puede concluir en lo que respecta al número de eosinófilos en la sangre periférica, y en concordancia con la vasta literatura al respecto que se encuentran elevados en los asmáticos, y en relación directa con el grado de positividad del test de alergia intradérmico, $(1,3,7)$.

\section{RESUMEN}

Se analizan 108 niños portadores de Asma bronquial perenne desde un punto de vista clínico y de laboratorio.

Se pone de manifiesto que si bien el asma bronquial es una afección que se inicia precozmente en la vida, hay una demora en plantearse el diagnóstico y ser referido a un centro especializado.

Se puede concluir la importancia de un sustrato atópico en el asma infantil por el alto porcentaje de antecedentes de alergia familiar $(72,2 \%)$, asma bronquial $(62 \%), y$ la alta incidencia en estos pacientes de afecciones bronquiales obstructivas en el ler. año de vida $(45,3 \%)$.

Queda demostrado la importancia de la infección respiratoria como factor desencadenante de las crisis asmáticas.

Se establece el alto porcentaje de positividad $(93,5 \%)$ del test intradérmico de alergia y la relación que existe entre la posibilidad de manifestar reacciones alérgicas a más de un alergeno. El asma "intrínseco" sería de escasa frecuencia en el niño.

Por último se plantea el alto \% de niños asmáticos con cifras elevadas de eosinófilos en sangre periférica y su relación directa con el grado de intensidad de las reacciones del test de alergia intradérmico.

\section{SUMMARY}

108 perennial bronchial asthmatic children are analized both from the clinical and laboratory point of view.

It is clear that, although bronchial asthma is an affection with an early beginning in life, there exist certain delay in its diagnosis, as well as in its reference to a specialized center.

Because of the high percentage of family allergy $(72,2 \%)$, bronchial asthma $(62 \%)$ and the high 
incidence of obstructive bronchial affections upon these patients $(45,3 \%)$ we must conclude about the importance of an atopic substrate in children's asthma during their firts year of life.

The high influence of the respiratory infection as a decisive factor during the crisis has been shown.

The intradermic allergy test is proved to be highly positive $(93,5 \%)$. The posibility to determine allergic reactions with more than one allergen is stablish. The "intrinsec" asthma is seldom ob. served in childhood.

Lastly it is stated that a high percentage of asthmatic children have a large amount of eosinophilies in peripheral blood, and a close relationship with its degree in their reactions to be intradermal allergic test.

\section{REFERENCIAS}

1.- Margaret Turner. "Asthma". Medicine 14: 889; 1972-1974.

2.- Frandsen S. "Bronchial Asthma among school Children in Copenhagen". Acta allergol. 12 : 341 ; 1958.

3.- Kendig E.L.; jr.: "Disorders of the Respiratory Tract in children". Philadelphia W.B. Saunders, 1967.

4.- Gómez, Gonzalo; Ibáñez, Sergio. "Aspectos etiopatogénicos del Asma bronquial en el niño". Rev. Chilena Pediatría. Vol. 45: 88, enero-Febrero, 1974.

5.- H. Mansmann, jr. "Management of the child with Bronchial Asthma". The Ped. Clinics of N.A. 15: 357, 1967.

6.- Hilman B.C. "The allergic Child" Ann of Allerg. 25: 620, 1967.

7.- Kantor $y$ Speer. "Characteristics of the allergic child". The Allergic child. Ed. por Speer, Hosbel, New York, 1963.

8.- Harnen, Kaas et al "IgE concentratione in allergic asthma in children". Arch of Disease in Child. 48: 850, 1973.

9.- L. Kunar; R.W. New-comb; K. Ishizaka; E. Middleton, Jr.; M.M. Hornbrook: "IgE levels in sera of children with asthma". Pediatrics. Vol. 47, № 5: 848; Mayo 1971.

10.- Lichtenstein and Margollis: "Histamine re. lease in vitro, Inhibition by cathecolamines and Methilxantines". Science; 161: 902, 1968.

11.- Frek, O. "Mediators of Atopic and anaphylactic Reactions", The Ped. Clin. of N.A. 16: 95, 1969.
12.- Pearlman, D. "Inmunoglobulins and allergic Diseases". The Ped. Clin. of N.A. 16: 118, 1969.

13.- Carrasco, E. y Col: "Mecanismos Inmunológicos en las enfermedades del Aparato respiratorio". Arch. Arg. de Alergia. 10: 16, 1973.

14.- Soerensen, k. y Col: "Enfermedades del aparato respiratorio, vistas por el inmunólogo". Rev. Chil. de Ped. 44: 362, 1973.

15.- Brent Taylor y Cols.: "Transient IgA deficiency and Pathogenesis of Infantile atopy". Lancet, Vol, 2: 111, 1973.

16.- Heimlich E.: "Asthmatic Hyperresponsiveness". The Ped. Clin. of N.A. 16: 149, 1969.

17.- Szentivanyi, A.: "The Beta Adrenergic Theory of the atopic Abnormality in Bronchial Asthma". J. of Allergic, 42: 203-232, 1968.

18.- Gellis Sydney, S.; Kagan. Benjamin M.: "Current Pediatric Therapy". 4 Philadelphia, W.E. Saunders. 1970.

19.- Smith and Parker: "The Responsivencss of Leukocyte ciclic AMP to adrenergic agents in pacients with asthma". Proc. Central Soc. Clin. Res. 43: 76, 1970.

20.- Mathison D.A.: "Clinical Profiles of Bronchial Asthma". JAMA, 224: 1134, 1973.

21.- R. Beltran, Oehling, "El factor inhalante y bacteriano en el asma infantil", Allerg. ct inmunopat. Vol. 1: 61, 1972.

22.- L. Tuft, H. Mueller; Alergia en el niño, Pág. 228. Editorial Pediátrica Barcelona, 1971. trics, Vol. 42: 1, 1969.

23.- Johnstone, Douglas, y Cols: "The value of Hyposensitization Therapy for bronchial Asthma un children. A 14 year Study". Pediatrics, Vol. 42: 1, 1969.

24.- Aas Kjell, "Alergic Asthma in childhood". Arch. Dis. Child. 44 : 1, 1969.

25.- Kenneth Mc Intosh; Elliot F. Ellis; Leonard, S. Hoffman; Tillinghast, G. Lybass, Jerry $y$ Eller, and Vincent A. Fulginatt. "The association of viral and bacterial respiratory infections with exacerbations of Wheezing in young Asthmatic children". The Journal of Pediatrics. Vol. 82: 578, 1973.

26.- Heinz y Wittig, C.M. Chang, "Bronchiolitis or Asthma". Ped. Clin. of N.A. 16: 55, 1969.

27.- Marie Britt, "Skin testing: Concepts and Realities". Med. Clin. of N.A. 16: 227, 1969. 